\title{
Verdad y atención al enfermo terminal
}

\section{(Truth telling and terminal patient care)}

\author{
Luís G. Soto y Carlos Sánchez Fernández de la Vega
}

Recibido: 10 de febrero de 2012

Aceptado: 16 de abril de 2013

\section{Resumen}

En el presente escrito, abordamos la cuestión de la verdad en la relación médico-paciente, concretamente en el caso de los enfermos terminales. Analizamos los problemas y actitudes acerca de decir la verdad cuando se trata de una enfermedad mortal, concluyendo lo importante que es que haya una comunicación fluida y veraz en la relación terapéutica. Así mismo, examinamos y destacamos el papel que pueden tener los médicos generales -el médico de cabecera- en la atención a enfermos terminales en el hogar y con sus familiares y/o amigos.

Palabras clave: decir la verdad, relación médico-paciente, médico general, atención a enfermos terminales, morir.

\begin{abstract}
The aim of this text is to examine the issue of truth telling in doctor-patient relationships, namely in the case of terminal patients. We analyze the problems and attitudes regarding truth telling that there are present when patients suffer from mortal diseases. We conclude that it is very important to keep a fluent and truthful communication in the doctor-patient relationship. We also examine and stress the role that general practitioners can play in the care of terminal patients at their home and with their friends and/or relatives.
\end{abstract}

Keywords: truth telling, doctor-patient relationships, general practitioners, care of terminal patients, dying. 


\section{Introducción}

En esta indagación conjunta sobre la verdad y la atención al enfermo terminal, partimos de nuestras experiencias profesionales como médico y como filósofo. Por un lado, la experiencia como médico general, durante décadas en el sistema sanitario público, la práctica del día a día y la reflexión sobre ella ${ }^{1}$. Por otro lado, la experiencia, también durante décadas, en la universidad pública, como docente e investigador en el campo de la ética. La bioética 2 nos proporcionó a ambos un lenguaje en el que entendernos, con el que mantener una conversación filosófico-científica en estos últimos años, fruto de la cual son las presentes reflexiones.

Otra aclaración más: abordamos la cuestión de la verdad como parte de la relación médico-paciente, una relación que, a nuestro entender, en el caso del enfermo terminal, debería llegar hasta el desenlace fatal. Insistiremos, no obstante, en la comunicación del diagnóstico, porque este momento concentra -y permite tratarlos principales aspectos de la problemática. Pero, desde ahora, recordamos que, después de un diagnóstico y un pronóstico mortal, la vida sigue, quizá no por demasiado tiempo o con un plazo más o menos limitado y, en cualquier caso, en unas condiciones muy distintas a la habitual normalidad cotidiana. $Y$ en ese lapso y trance, en ese espacio-tiempo, puede tener un papel relevante el médico general, junto con los familiares y allegados que eventualmente puedan acompañar al enfermo.

\section{Decir la verdad}

Aunque se trata de algo resuelto desde el punto de vista legal ${ }^{3}$, comunicar un diagnóstico fatal a un paciente es una cuestión problemática, que suscita controversia. ¿Qué hacer? Con frecuencia, incluso entre los profesionales, surgen dudas acerca de la conveniencia de decir la verdad al enfermo.

\footnotetext{
1 Carlos Sánchez Fernández de la Vega: "Helping The Terminal Patients In The End Of Their Life", International Association of Gerontology and Geriatrics: VI European Congress, 5-8 July 2007, Saint Petersburg, Russia; "Bioethics and The Terminal Patient Care", 18th WONCA World Conference, 2427 July 2007, Singapore. En estas intervenciones, se consignaban los resultados satisfactorios obtenidos en catorce casos.

2 En especial y en concreto: Hottois \& Missa 2005.

3 Ley 41/2002, de 14 de noviembre, básica reguladora de la autonomía del paciente y de derechos y obligaciones en materia de información y documentación clínica. Igualmente, la legislación autonómica, por ejemplo: Llei 21/2000, de 29 de desembre, sobre els drets d'informació concernent la salut i l'autonomia del pacient, i la documentació clínica, en Cataluña; Lei 3/2001, de 28 de maio, reguladora do consentimento informado e da historia clínica dos pacientes, Lei 3/2005, de 7 de marzo, de modificación da Lei 3/2001, de 28 de maio, reguladora do consentimento informado e da historia clínica dos pacientes, en Galicia; etc. Una aproximación sintética al marco jurídico y deontológico en España relativo a la obligación de informar: Simón \& Júdez 2004, pp. 39-43.
} 


\subsection{El principio de autonomía: la regla}

Sin embargo, desde el punto de vista ético tampoco parece haber dudas. El principialismo, la metodología bioética más extendida y de la que Beauchamp y Childress ofrecen una formulación canónica y llena de matices ${ }^{4}$, dice -coincidiendo en esto con la legalidad- que en estos casos debe prevalecer el principio de autonomía, que obliga a decir la verdad al paciente, sobre los principios de beneficencia y de no-maleficencia, que podrían excusar ese deber pensando en, con ello, hacer un bien o, por lo menos, evitar un mal -un sufrimiento, incluso un daño, psicológico- al enfermo.

¿Por qué debe el facultativo decir la verdad y comunicar el diagnóstico y pronóstico al paciente? Porque este, para poder decidir, en lo que respecta a su salud y en general a su vida, tiene que tener conocimiento de su situación. Solo así podrá otorgar un consentimiento informado a una posible intervención o, al contrario, recusar el tratamiento propuesto. $\mathrm{Y}$, además, el enfermo podría tomar las decisiones que estime oportunas para enfrentar una perspectiva vital con un horizonte final señalado.

Vistas así las cosas, el asunto parece resuelto... y, sin embargo, no lo está, o no del todo. Hemos dicho que el paciente podrá decidir, que el enfermo podrá tomar las decisiones oportunas, pero: ¿estará verdaderamente en condiciones de hacerlo? De hecho, resulta frecuente pensar que una persona, tras la noticia y bajo el impacto de saberse en enfermedad terminal, no se encontrará probablemente en condiciones de afrontar esa situación y resolver las cuestiones que se le presenten. No todas las personas reaccionan igual y, según se insiste, muchas preferirían no saber nada.

\subsection{El principio de beneficencia: las excepciones}

Ciertamente, no es difícil imaginar situaciones de gran vulnerabilidad y/o de fragilidad en las que la comunicación de la noticia - decir la verdad - perjudicaría y/o agravaría el estado del paciente. Cuando pensamos así, lo que estamos haciendo es tener en cuenta y dar prioridad a los principios de no-maleficencia y beneficencia... que en esos casos de vulnerabilidad y fragilidad podrían prevalecer sobre el principio de autonomía. Contemplamos -admitimos, pues- excepciones a la regla general que sigue siendo decir la verdad 5 .

\footnotetext{
${ }^{4}$ Beauchamp \& Childress 2002. Es traducción de la edición de 1994. La quinta, de 2001, no contiene variaciones por lo que respecta a la formulación del principialismo (sí, en cambio, acerca del marco moral en que se inscribe).

5 "In summary, there are some circumstances in which the health professions are probably exempted from society's general requirement for truthfulness. But not telling the truth is usually the same as telling a lie, and a lie requires strong justification. Lying must be a last resort, and we should act as if
} 
Ahora bien, la actitud espontánea, e incluso reflexiva, tiende a convertir esas excepciones en la regla general. Si preguntamos a alguien qué haría -qué se debe hacer - en esa situación e incluso qué preferiría si estuviese en ese caso, probablemente optaría por la información indirecta, a familiares y/o allegados, y, de ser el afectado, quizá preferiría no saberlo, invocando para justificar esta actitud un derecho a no saber. De hecho, esas son respuestas que aparecen cuando, sin proporcionar una formación previa y antes de entrar en análisis detallados, les planteamos este problema a los estudiantes 6 . Inclusive, después de analizar y debatir el asunto, mostrando lo que marca la legalidad y las pautas dominantes en la bioética, siempre hay quien persiste en defender el derecho a no saber.

\section{No querer saber}

¿De dónde viene, en qué se asienta esa resistencia a decir/saber la verdad, cuando esta es el pronóstico de un desenlace fatal? En nuestra opinión, esta denegación de la verdad tiene raíces -causas- psico-sociales, es decir, psicológicas y sociales que están interrelacionadas.

\subsection{Carencias vitales personales}

En primer lugar, hemos de reconocer que, por lo general, el individuo -una persona - carece de recursos para afrontar psicológicamente una enfermedad mortal y un desenlace fatal. En efecto, en nuestra sociedad occidental contemporánea el individuo - por lo general, repetimos - carece de formación y de experiencia para enfrentarse con la muerte. Afortunadamente, en nuestra sociedad, casi todos filosofamos un poco, pero escasea - por no decir que falta - la formación necesaria para encarar la muerte (la de los demás y la propia)... que es un aspecto ineludible de la vida.

Ahí tocamos la otra carencia: la pobreza de la experiencia. No se disfruta, por lo general, de una vida satisfactoria, sino de estrecheces y limitaciones ${ }^{7}$. Las personas no suelen gozar durante su vida de la autonomía, la capacidad de decidir y de

\footnotetext{
we were to be called upon to defend the decision in public debate, even if our duty of confidentiality does not allow this in practice. We should always aim to respect the other important principles governing interactions with patients, specially the preservation of the patient's autonomy" (Higgs 2001, p. 92).

6 En concreto, presentamos un supuesto general formulado por Marie-Hélène Parizeau (Parizeau 2005, p. 908) a estudiantes de postgrado titulados en psicología (Máster de Psicoxerontoloxía, Facultade de Psicoloxía, Universidade de Santiago de Compostela, cursos 2010-11, 2011-12 y 2012-13).

7 Cfr. "La vida buena. Un marco político justo y universal. La mística natural y el sentido de la vida. El silencio" (Sádaba 2009, pp. 249-266).
} 
hacer,... que luego se espera que muestren ante la muerte: viviendo en la zozobra resulta difícil afrontar el morir con serenidad. En suma, la falta de filosofía ${ }^{8}$ y la precariedad de la existencia personales endurecen el trance de la enfermedad mortal... en nuestro contexto actual, la sociedad occidental contemporánea. Esta precisión es necesaria, pues con menos filosofía y más precariedad en otros tiempos y otros lugares no resulta tan duro -no les resulta tan duro a los individuos - el trance de morir.

\subsection{Déficits culturales sociales}

En efecto, en segundo lugar, nuestra cultura moderna, el mundo en el que vivimos, tiende a reprimir la muerte, a ocultarla como hecho social. Ariès y otros lo han venido señalando desde el último tercio del siglo XX. Hasta hace poco, y aún ahora aquí al lado -en Galicia y, también, en España - en localidades pequeñas podemos verlo, la cultura vigente trataba de otro modo la enfermedad y la muerte ${ }^{9}$. Esta en la cultura tradicional es algo visible, y bien iluminado, ocupa un lugar central y otorga al individuo, al enfermo y moribundo, un protagonismo social. Aún hoy, en pueblos y aldeas, la enfermedad y la muerte, y los rituales de esta, son un lugar de encuentro y de vida social, de reunión y acción colectivas ${ }^{10}$. Quien muere se singulariza y protagoniza un acontecimiento que concita el interés y la movilización de la comunidad. Enfermar y morir son actos sociales.

No sucede lo mismo en nuestra cultura moderna, que hace una vigorosa promoción (también publicitaria, en gran medida ideológica) de la salud y la vida, y en la cual la enfermedad, en especial la enfermedad mortal, y la muerte ocupan un lugar marginal.

Nuestra cultura moderna tiende a hacerlas invisibles, intenta hacerlas desaparecer11. La muerte (la enfermedad, el sufrimiento, etc.) pone en cuestión -en jaque, podríamos decir - el sentido de la vida, es decir, el valor que para una persona puedan tener las ofertas que nuestra sociedad nos brinda y las obligaciones que esta nos

\footnotetext{
$8 \mathrm{Al}$ contrario, el conocimiento y la frecuentación del pensamiento filosófico pueden ayudar a plantear cuestiones y resolver problemas psico-sociales. Así lo ha hecho siempre la fillosofía, pero a escala minoritaria, entre aquellos pocos que la han tenido a su alcance. A comienzos del siglo XXI, la función "terapéutica" de la filosofía ha conocido una popularización exitosa con el título de Marinoff que es casi un emblema: Más Platón y menos prozac (Marinoff 2003). Hoy día, en nuestro pagos, resulta difícil preguntarse y hallar respuesta acerca del sentido de la vida, aún cuando se abrace una opción religiosa, sin recurrir a la filosofía. El sentido de la vida es una cuestión crucial cuando se trata de encarar la muerte. Vid. "Las respuestas para superar el sinsentido de la vida" (Sádaba 2012, pp. 5574).

9 En palabras de Ariès, en la cultura europea tradicional -desde el medievo hasta hace relativamente poco - la muerte está "domesticada". Vid. "La mort apprivoisée" (Ariès 1975, pp. 21-35).

10 Vid. Gondar 1989.

11 "La mort interdite" (Ariès 1975, pp. 67-78).
} 
impone. Todo pierde valor ante la muerte: nuestra sociedad intenta vencerla por todos los medios (movilizando sus recursos científico-técnicos, políticos, económicos, etc.)... y, cuando no puede hacerlo, opta por ocultarla. A pesar de los avances en bienestar en los últimos dos siglos, nuestra civilización científico-técnica, así como nuestra organización política y económica, no pueden asegurar una vida digna y duradera a todos (ni siquiera a muchos), por lo que han optado por ocultar la presencia de la muerte, su cotidianidad, la amenaza constante que representa, la elevada frecuencia con que aparece.

Morirse, enfermar y morir, ya no es ser protagonista de un hecho social ${ }^{12}$, sino entrar en una zona de sombra, caerse de la vida social, de sus rutinas, sus pautas y sus ritos. Enfermar mortalmente es entrar en un espacio y un tiempo sin sentido, preludio -e inicio ya- del abandono de la vida. El enfermo terminal no tiene un papel en nuestra cultura, en nuestro contexto social: queda desubicado, no sabe qué hacer, no se espera nada de él. Esta cultura y esta sociedad nuestras no le dan, no le ofrecen, recursos (pautas, apoyos, costumbres) para afrontar la situación en la que se encuentra. Y, además, han convertido la muerte en un tabú, lo que dificulta la comunicación y el diálogo entre el médico y el paciente: no solo la comunicación del diagnóstico y pronóstico, sino el diálogo posible en el curso de la enfermedad.

\section{La verdad en el contexto y en el interior de la relación terapéutica}

Esta profunda etiología cultural-social de la resistencia a decir/saber la verdad cuando se trata de una enfermedad mortal suele pasar desapercibida, insistiéndose en aspectos que conciernen a los actores de la situación, fundamentalmente, médico y paciente. Las razones invocadas para no decir y para no saber pueden remitirse al beneficio del enfermo: no querer causarle daño ${ }^{13}$, no dañar la relación terapéutica, evitar sufrir más y con ello estar aún peor.

\subsection{El punto de vista médico}

Así, los profesionales señalan a veces que un pronóstico fatal, un diagnóstico ante el cual no hay nada -o casi nada - que hacer, significa reconocer el fracaso de la medicina o, dicho en términos más generales, de la ciencia. El fracaso daña la imagen de la ciencia y ocasiona una pérdida de poder de la misma, que resulta visiblemente desautorizada por los hechos. Por esta razón, nuestra civilización científico-técnica tiende a ocultar la muerte: porque muestra la fragilidad y la vulnerabili-

12 "La mort inversée. Le changement des attitudes devant la mort dans les sociétés occidentales", en concreto lo relativo a "comment le mourant est privé de sa mort" (Ariès 1975, pp. 180-189).

13 "Primum non nocere. O concepto de "dano" e os seus problemas" (Gracia 2008, pp. 5-17). 
dad de aquellos poderes -la ciencia y la técnica, mas también la economía y la política - que pretenden organizar la vida de las personas y las sociedades. De un modo similar, podríamos afirmar que el fracaso de la medicina, ante la enfermedad mortal, daña el poder médico, impotente a ojos del paciente para revertir el curso de los acontecimientos.

Sin embargo, no es esta -o no es exactamente así- la preocupación que hemos encontrado en nuestra experiencia. La razón que los profesionales esgrimen para no decir la verdad, o no decirla por completo, no es retener un poder suyo, personal y/o institucional, sino mantener la confianza del paciente, necesaria para el funcionamiento de la relación terapéutica. En suma, de lo que se trataría sería de evitar la pérdida de la confianza que debe existir en la relación terapéutica, con lo que, en última instancia, se intentaría proteger el beneficio del paciente (por lo menos, ahorrándole perjuicios).

Un testimonio reciente viene en apoyo de nuestras reflexiones y nuestra experiencia: Winokur ${ }^{14}$, un oncólogo retirado, señala esas dos razones y añade una tercera, atinente a la medicina privada: el temor a perder al paciente, a que se vaya a otro médico ${ }^{15}$. De las dos razones aducidas, considera la más común -la ofrecida por la mayoría de los oncólogos- no querer minar la esperanza del paciente y protegerlo de sus propias reacciones, que lo podrían llevar a descuidar o incluso abandonar el tratamiento. Sin embargo, en su opinión (y por lo que a él respecta), la razón última es la primera: no querer reconocer que los conocimientos y habilidades médicas son limitados. En suma, el facultativo se resiste a informar verazmente también por este motivo... y no solo porque piensa que el paciente se resiste a saber la verdad y es mejor para el enfermo no decírsela.

\subsection{Un marco nuevo médico-paciente}

Esta actitud paternalista no encaja bien en un modelo de relación médicopaciente que ya no es paternalista. En el curso del siglo XX en el mundo occidental, la relación terapéutica dejó de funcionar y regirse según un modelo jerárquicovertical paternalista, para hacerlo siguiendo un modelo comunicativo-horizontal contractual. Otrora, el médico se encontraba en un plano superior con respecto al paciente, que prácticamente carecía de posibilidades de exigirle responsabilidad por sus actuaciones, salvo que estas entrasen en el cuadro delictivo (por ejemplo, el envenenamiento). El médico no compartía su poder-saber con el enfermo, que literalmente se ponía en las manos de aquel. El médico tenía entonces un poder pasto-

\footnotetext{
14 Winokur 2012: "Why We Avoid Telling Patients the Truth", publicado en The Health Care Blog el 18/08/2012. Poco después apareció traducido en Diario Médico, por cuya edición citaremos. El texto original puede consultarse en http://thehealthcareblog.com/blog/2012/08/18.
}

15 Winokur 2012, p. 2. 
ral, semejante al de un sacerdote ${ }^{16} \mathrm{y}$, en términos políticos, al de un líder carismático o al de un monarca absoluto. En suma, el facultativo poseía, a su escala particular y en el ámbito de su actuación, un poder semejante a los imperantes otrora en la sociedad.

Hoy por hoy, aquí y ahora, nos encontramos en un panorama completamente diferente: no solo la sociedad ha cambiado, también lo han hecho la profesión y la práctica médicas. En la segunda mitad del siglo XX, la relación médico-paciente17 se ha ido entendiendo, cada vez más, como una relación contractual, tomando como referencia el trato privado de prestación de un servicio teniendo como contrapartida una retribución. Y como tal contrato, la relación médico-paciente da lugar a una responsabilidad que se puede exigir en términos jurídicos, en sede judicial. O sea, el médico que no recibe cobro puede demandar al paciente,... mas también este puede reclamar al facultativo indemnización por la prestación recibida, por los defectos habidos en esta y los daños y perjuicios en consecuencia ocasionados.

\subsection{Contractualización, contractualismo}

Esta contractualización ${ }^{18}$-originariamente propia de la medicina privada- ha llevado a modificar las posiciones del médico y el paciente en la relación terapéutica, de la que ambos - en cierto modo- han pasado a ser sujetos y no, como en el modelo paternalista, uno sujeto, el facultativo, y el otro objeto, el enfermo. Este, al ir exigiendo responsabilidades por el tratamiento, se ha visto obligado a asumir responsabilidades en el tratamiento: a otorgar su consentimiento informado. Ciertamente, esa exigencia -dar o no el consentimiento al acto médico- es una muestra de respeto a la voluntad y a la persona del paciente ${ }^{19}$, que no puede ser tratado como una cosa.

Pero el consentimiento informado es también un mecanismo para salvaguardar la intervención del médico y limitar la responsabilidad en que pudiere incurrir. En

\footnotetext{
16 Sobre "religious foundations of trust in medicine": Imber 2008, pp. 3-42.

17 "Metaphors and Models of Doctor-Patient Relationships: Their Implications for Autonomy" (Childress \& Siegler 2001, pp. 71-80). Estas metáforas y modelos nos dan una idea de la naturaleza y la riqueza de la relación terapéutica, establecida en un marco contractual pero no reducible a un contrato. Por ejemplo, el sentimiento puede (y según algunos debe) tener un papel en la relación clínica (Pose 2009, pp. 50-57).

18 Está relacionada con otras transformaciones experimentadas por la medicina en Occidente desde comienzos del siglo XX: generalización (difusión de la medicina y la oferta médica por todo el cuerpo social), mercantilización (equiparación del ejercicio de la medicina al trabajo de otros profesionales), especialización (desplazamiento del médico general, como figura tipo del facultativo, por el especialista). Cfr. Imber 2008, pp. 175-178.

19 Es menester respetar la voluntad de la persona inclusive, según algunos, cuando el paciente en su decisión se equivoca de modo flagrante: "El derecho a que nos dejen en paz" (Engelhardt 1995, pp. 327-330).
} 
este punto, el interés del facultativo y el interés del paciente coinciden en privilegiar el principio de autonomía sobre el principio de beneficencia. Aunque no siempre se vea así: aunque no siempre el médico o el paciente lo vean así. Este porque no quiera saber, prefiera "pasar": inhibirse y/o delegar, dejar pasar y/o dejarse llevar, etc. El médico porque cree ponerse en el lugar del paciente, pensando que el bien de este pasa por renunciar a la autonomía. Sin embargo, esta renuncia en términos legales solo es admisible en condiciones establecidas.

Ahora bien, lo que ha desplazado al paternalismo, más que el contrato, es el contractualismo: el contractualismo como marco moral en el que tiene lugar la relación terapéutica, como una de tantas -una más- de las interacciones interpersonales posibles en nuestra sociedad. Es decir, no solo se ha instaurado el contrato en términos jurídicos en el seno de una relación anteriormente apenas regulada, sino que esta relación -convertida ahora en una inter-relación- se inscribe en un marco contractualista moral: en un contexto de entendimiento y cooperación entre agentes que obran buscando el beneficio mutuo y considerando el bienestar general. Esto significa que en la relación terapéutica, más allá (y más acá) de los términos jurídicos, existe un compromiso moral relativo a la expresión y comunicación de expectativas y pretensiones, al cumplimiento de obligaciones, fidelidad a las promesas, lealtad a las personas, etc.

En suma, no podemos -no debemos- contemplar la relación médico-paciente como un contrato "solo" legal, sino también, y fundamentalmente, moral. Debemos verlo -pensamos-como expresión y consecuencia -como una form-del contractualismo moral, difuso pero extendido, inclusive prevaleciente como actitud moral, en las sociedades democráticas occidentales contemporáneas ${ }^{20}$.

\subsection{Paternalismo: acepciones, salvedades}

Por otra parte, también hay que introducir matices en la definición, y hacer salvedades en la valoración, del paternalismo. De hecho, Beauchamp y Childress, recogiendo entre otros los aportes de Feinberg y de Dworkin, ya ofrecen una visión matizada del paternalismo, así como de su justificación y aceptabilidad ocasionales ${ }^{21}$. En línea con Feinberg, distinguen entre una versión débil (o blanda) y una fuerte (o dura), y como este y como Dworkin vinculan su aceptabilidad al consentimiento de los beneficiarios. Si hay o puede haber consentimiento, el paternalismo es débil (o blando) y aceptable. E incluso en algunas circunstancias excepcionales puede ser aceptable el paternalismo fuerte, la acción benefactora en contra de las preferencias expresas o conocidas del beneficiario. ¿Será ese el caso de la verdad en la comunicación con el enfermo grave y/o terminal? Pensamos que no, aunque

20 Vid. "La concepción plausible de la moral" (Tugendhat 2010, pp. 76-93).

${ }^{21}$ Beauchamp \& Childress 2002, pp. 301-309. 
creemos que en tal situación debe revalorizarse el paternalismo. Comenzaremos por intentar enfocarlo más adecuadamente.

Más recientemente, siguiendo la estela de Feinberg, Beauchamp y Childress, y Dworkin, Nilstun ha precisado distinguiendo, además de entre paternalismo débil y fuerte, entre paternalismo pasivo y activo ${ }^{22}$. Asíi ${ }^{23}$, distingue cuando el beneficiario consiente (prefiere, desea) o no que se produzca una interferencia en su autonomía, siendo el primero paternalismo pasivo y el segundo paternalismo activo. $Y$ diferencia, además, si el beneficiario es autónomo (paternalismo fuerte) o no lo es (paternalismo débil). Obviamente, el pasivo y el débil pueden resultar bastante aceptables. Y, apuntamos por nuestra parte, la admisión de la interferencia del terapeuta y el devenir no-autónomo (como mínimo, menos autónomo) del paciente son circunstancias bastante probables en la enfermedad terminal.

Una última idea con respecto al paternalismo: habitualmente lo entendemos a partir de sus formulaciones políticas, además por lo general autoritarias, que merecen un amplio rechazo ${ }^{24}$. Pensémoslo a partir del modelo de la familia, no como poder paternal, sino como acción parental, relativa al cuidado y la crianza de los hijos por parte del padre y/o la madre. Obviamente, esta acción benefactora, ampliamente aceptada, debe cesar cuando choca con la autonomía de los beneficiarios. Y no olvidemos, además, que la relación y acción parentales están sujetas a regulación jurídica y moral. Sin caer en idealismos, el paternalismo así contemplado -visto en la perspectiva del cuidado - ofrece un ángulo, incompleto mas no inapropiado, para comprender la relación terapéutica en situación terminal.

En resumidas cuentas, y dicho con otras palabras, un "principio de paternalismo" puede funcionar como complemento del principio de autonomía 25 .

\subsection{Veracidad, responsabilidad}

Decir la verdad al paciente constituye, en la actualidad, un deber no solo moral sino también legal26.

Moralmente, la verdad es considerada un ingrediente necesario, fundamental, para la existencia y desarrollo de las relaciones humanas: sin ella, la vida social

\footnotetext{
22 También entre paternalismo social e individual, según el beneficiario sea un grupo o un individuo (Nilstun 2005, p. 685).

23 Vid. Nilstun 2005, pp. 685-686.

24 Desde esta perspectiva social, Mill sienta en On Liberty, en 1859, las bases del anti-paternalismo. Pero también indica los supuestos en que la acción -la intervención, la regulación- paternalista sería aceptable. Vid. Mill 1981.

25 Vid. Lora \& Gascón 2008, pp. 49-50.

26 Se trata, sin embargo, de una obligación reciente, con escasa tradición en la historia de la medicina: desde sus orígenes, la deontología médica ha venido ignorando el deber y las "virtudes" de la veracidad (Beauchamp \& Childress 2002, pp. 425-426).
} 
peligra $^{27}$. En el contexto terapéutico, la veracidad es fundamental para generar confianza y asegurar la fidelidad en la interacción. Debe haber confianza entre el facultativo y el enfermo, así como la seguridad de que uno y otro cumplirán los compromisos adquiridos. La veracidad es necesaria para una relación terapéutica fructífera. Obviamente, hay excepciones y, además, matices, pues, en lo que respecta a faltar a la verdad, no es lo mismo la mentira que la reserva y que la omisión. Mas, como principio general, el facultativo tiene la obligación de decir la verdad. Y este deber moral va acompañado de una obligación legal. Cosa muy a tener en cuenta al considerar las posibles excepciones del deber de informar verazmente, pues lo que puede parecer moralmente admisible (omisión, reserva, mentira... en ciertos casos) puede resultar jurídicamente punible.

En efecto, no decir la verdad vicia y falsea cualquier contrato y, por lo tanto, también el contrato médico-paciente. En consecuencia, de no decir la verdad pueden seguirse responsabilidades para el médico, no solo en el ámbito de la salud. El enfermo, en efecto, puede resultar perjudicado por no conocer su situación y no poder tomar las decisiones que estimare oportunas, no solo con relación a su salud, sino también a su patrimonio, cosas que, con frecuencia, también implican a terceros, que se pueden ver perjudicados o beneficiados por la situación. Y, en consecuencia, estos terceros también podrán encausar al facultativo.

\subsection{La perspectiva del enfermo}

En cuanto al paciente, no debemos pensar que es universal el deseo de no saber, que todos los enfermos querrían ignorar su estado. De hecho, cuando sometemos a la consideración de los estudiantes esta cuestión, prevalece querer saber y el deber de informar.

Así, cuando preguntamos a los estudiantes qué preferirían en esa disyuntiva, pugnan dos actitudes: querer saber, para poder decidir; preferir no saber, para vivir - estar, morir- lo más tranquilo posible. Y, aunque siempre hay alguien que defiende no saber, tiende a prevalecer abrumadoramente 28 querer saber, formulándose esta exigencia como derecho a saber, como parte imprescindible del derecho a decidir sobre la vida propia. De todas maneras, estas respuestas son indicativas de lo que se piensa que se debería hacer en esa situación. Y, probablemente, de encontrarse efectivamente cada uno en tal situación, las actitudes variarían. Parece razonable

\footnotetext{
27 En ello, la filosofía ha insistido e insiste. Constituye una referencia fundamental la argumentación que ofrece Kant, en 1785, en la Grundlegung zur Metaphyisik der Sitten, BA 54-55 (Kant 1988, pp. 60-61). Tugendhat matiza y actualiza esa argumentación (Tugendhat 2010, pp. 140-142). Si lo despojamos de su exageración, también resulta fructífero a nuestros efectos el escrito "Sobre un presunto derecho a mentir por filantropía" (Kant 1986, pp. 61-68).

28 Más del 90\%, tomados en conjunto los datos de 2010-11, 2011-12 y 2012-13.
} 
pensar que aumentaría el rechazo a saber, pues la denegación es una respuesta psicológica 29 no infrecuente ante la adversidad, y que, cuando menos, aumentarían las dificultades psicológicas de la persona afectada para encarar efectivamente una enfermedad mortal con pronóstico fatal.

Curiosamente, cuando pedimos a los estudiantes que adopten la perspectiva del informador, aumentan las reticencias y los matices. Los estudiantes apuntan variables que aconsejan limitar e incluso suprimir la información: la edad, el estado psíquico, la situación personal y/o familiar del enfermo, fundamentalmente. De todas las maneras, a pesar de señalar razones para no informar, el balance es netamente favorable a las razones para informar ${ }^{30}$. La cuestión que surge es cómo hacerlo. Y ahí otro dato curioso: los estudiantes suelen insistir en la necesidad de trabajar con el facultativo, antes que con el enfermo.

Este, ciertamente, teme la información, pero no cabe sostener que los pacientes rechazan conocer la verdad. Simplificando, podemos decir que lo que se teme son las consecuencias de esa información, lo que significa en términos psico-sociales convertirse en un enfermo sin curación y encaminarse a la muerte ${ }^{31}$. Numerosos estudios y opiniones coinciden en señalar que lo que el paciente teme, en esa situación en la que aumentan su fragilidad y vulnerabilidad, es el abandono y la soledad: la falta de la atención y el acompañamiento necesarios para poder vivir hasta el final ${ }^{32}$. Según estudios recientes ${ }^{33}$, la verdad no mina la esperanza del paciente: al contrario, facilita la relación con los cuidadores. Lo que el paciente necesita es saber que va a contar en todo trance con esa atención médica. Que esta le va a permitir enfrentarse al dolor y el sufrimiento, y que podrá disponer de la cantidad y calidad de vida, las máximas en su situación, para satisfacer las obligaciones y deseos que estime más importantes. Obviamente, esta actitud valiente resulta favorecida si se da un acompañamiento por parte de allegados o familiares. Porque, como decíamos al principio, las circunstancias psico-sociales para enfrentarse con la enfermedad mortal son adversas. Y refuerzan el deseo de no saber, la actitud denegatoria en el paciente.

Sin embargo, aunque resulta comprensible el deseo de no saber, no es admisible un derecho a no saber. En términos generales, no cabe admitir, ni moral ni jurídicamente, que una persona renuncie a su autonomía y prefiera ignorar informacio-

\footnotetext{
29 Sobre las diferentes respuestas psicológicas: Doka 2010, pp. 120-150.

30 Razones para no informar y, más fuertes, para informar: Cabodevilla 2002, pp. 63-65.

31 Gil 2005, pp. 291-295.

32 Marques 2010, p. 150. Según este autor resume, lo que el paciente demanda en la enfermedad mortal y en el tramo final de la vida es "capacitação, dignidade, integridade" (Marques 2010, p. 149). Puede consultarse también en http://hdl.handle.net/10400.21/735.

33 Winokur 2012, p. 2: refiere un análisis publicado en The Journal of the American Medical Association y una investigación realizada en el VCU Massey Cancer Center.
} 
nes relevantes, pudiéndose seguir de ello perjuicios para ella misma o para terceros. Si admitiésemos el derecho a no saber, esos perjuicios quedarían amparados por la renuncia del sujeto a su capacidad de decidir y a su propio bien. Por esta vía, el sujeto consentiría el mal recibido en su persona o bienes y/o ocasionado a terceros, lo que exculparía a los causantes del daño. En términos generales, insistimos, el derecho a no saber no resulta admisible en el contexto de la relación terapéutica, pues satisfacer el deseo de no saber del paciente, respetar su rechazo a saber, se convierte en ocultación de información por parte del médico, incurriendo este en la responsabilidad subsiguiente. Decimos "en términos generales" porque la veracidad se relaciona con otras problemáticas, como la privacidad y la confidencialidad, que exigen realizar matizaciones. Y, por otra parte, el paciente puede "no entrar" o "no seguir" en la relación terapéutica: puede decidir ignorar el diagnóstico, no iniciar o abandonar un tratamiento.

\section{Información, comunicación}

Según los resultados de una investigación realizada en Málaga en el último lustro del siglo $\mathrm{XX}^{34}$, la información proporcionada por los médicos a los pacientes, inclusive en lo relativo al diagnóstico y pronóstico, era muy poca, prácticamente nula en una gran proporción, más o menos entre el $80 \%$ y el $90 \%$ de los $\operatorname{casos}^{35}$. Inclusive, en la fase terminal, el conocimiento del enfermo sobre su enfermedad y su situación, estaba en torno al 40\%36. Por el contrario, la información dada a los familiares y su conocimiento de estos ítems se situaba por encima del $90 \% 37$. Para evaluar correctamente estos datos, hay que tener en cuenta que el 75\% de los fallecimientos se produjeron en hospital 38 , que la mayoría de los médicos informantes son especialistas y solo un $20 \%$ médico de cabecera ${ }^{39}$, que el $60 \%$ de los facultativos tiene una relación con el paciente de un mes o menos 40 .

Traemos a colación estos datos porque, quizá no siendo representativos, probablemente siguen siendo significativos. Sin duda, la situación ha variado en esta primera década del siglo XXI, debido a la presión legal y también ética, pues la legislación y los comités y las comisiones han marcado el deber de informar. Mas, aunque la situación haya cambiado, pensamos que el problema persiste. Por ejemplo, y

\footnotetext{
34 Díez Ripollés, Santos Amaya \& Garrido de los Santos 2000.

35 Díez Ripollés, Santos Amaya \& Garrido de los Santos 2000, pp. 63-74.

36 Díez Ripollés, Santos Amaya \& Garrido de los Santos 2000, pp. 74-77.

37 Díez Ripollés, Santos Amaya \& Garrido de los Santos 2000, pp. 80-85.

38 Díez Ripollés, Santos Amaya \& Garrido de los Santos 2000, p. 40.

39 Díez Ripollés, Santos Amaya \& Garrido de los Santos 2000, p. 32.

40 Díez Ripollés, Santos Amaya \& Garrido de los Santos 2000, p. 33.
} 
de modo eminente, en EE.UU. la práctica de informar dio un vuelco de los años 60 a los años 80 del siglo pasado: en 1961, según un estudio, el 88\% de los médicos evitaba revelar el diagnóstico de cáncer; en 1979, según otro estudio, el 98\% de los médicos proporcionaba información del diagnóstico de cáncer ${ }^{41}$. Sin embargo, testimonios recientes muestran que el problema persiste: "ahora, años más tarde, el asunto no ha cambiado mucho. La mayoría de los enfermos con cáncer nunca reciben información de sus médicos acerca de su pronóstico o incluso de la muerte inminente"42. Quizá el porcentaje no sea tan elevado -"la mayoría"- como afirma este oncólogo estadounidense retirado. Pero su testimonio apunta a que el problema sigue ahí. Que está ahí, como en nuestro caso, en nuestros pagos ${ }^{43}$.

En definitiva, hay que informar, pero ¿cómo hacerlo? Según la opinión general, es recomendable -resulta casi obligado - modular la información, presentarla de la manera más conveniente y aceptable para el paciente. Cabe precisar algo más, señalando un umbral mínimo y un grado óptimo 44 .

El umbral mínimo lo representa el consentimiento informado, obviamente siempre que se trate de sujetos capaces y no se esté ante los supuestos excepcionales previstos por la legislación. El paciente debe tener la información básica para otorgar un consentimiento informado a las intervenciones a que hubiere lugar o, si no hay nada o casi nada que hacer, para que conozca la gravedad de su estado. Lo habitual y deseable es que esa información básica pueda completarse con otra más extensa o detallada a familiares y/o allegados. El umbral mínimo no es poco y se convierte en un máximo si en el consentimiento informado incluimos, como deberíamos, la sedación paliativa y/o terminal ${ }^{45}$. El grado óptimo incluye la posibilidad de una planificación anticipada de los cuidados, en colaboración con el paciente que otorga unas instrucciones previas 46 .

Todo esto resulta más factible cuando se trata del médico de cabecera -un médico general - que atiende a un paciente habitual. En ese caso, más que de información al enfermo, podremos quizá hablar de comunicación con el enfermo.

\footnotetext{
${ }^{41}$ Beauchamp \& Childress 2002, p. 429. Refiriéndose a 2001, Parizeau refrenda esos datos afirmando que en América del Norte (EE.UU. y Canadá) la práctica habitual es informar, salvo excepciones (cuando existe peligro de suicidio en el paciente), mientras que en Europa sucede lo contrario: suele ocultarse el diagnóstico de cáncer (Parizeau 2005, p. 908).

42 Winokur 2012, p. 2.

43 Otro ejemplo: en Portugal, en 2008, cerca de la mitad de los enfermos en fase terminal eran engañados (Marques 2010, p. 150).

44 Apoyamos nuestras reflexiones en Os coidados ao final da vida. Documento de recomendacións (Comisión Galega de Bioética 2012).

45 Comisión Galega de Bioética 2012, p. 49.

46 Comisión Galega de Bioética 2012, pp. 39-40.
} 


\section{Comunicación, relación, atención}

Además, decir la verdad no es algo que se reduzca a un momento puntual, la comunicación de un diagnóstico, sino que se extiende a lo largo de toda la relación terapéutica ${ }^{47}$. Puede reducirse a un momento puntual, cuando se consulta a un especialista. Mas, por lo general, la comunicación dura lo que dura la relación terapéutica. Y esta, en el caso del médico de cabecera, va más allá, comienza antes y sigue después, de lo que dura una intervención o un tratamiento. En nuestra opinión, el médico general reúne las mejores condiciones para administrar la verdad y atender al enfermo terminal.

\subsection{Atención médica y acompañamiento al enfermo}

Enfrentarse a una enfermedad mortal 48 es un trance muy difícil, que suele ser mejor no afrontar en soledad y con la exclusiva atención del personal sanitario49. Los familiares y/o allegados pueden cumplir un papel de acompañamiento difícilmente mejorable y difícilmente sustituible ${ }^{50}$. Y dentro del personal sanitario apuntamos el papel que puede jugar el médico de cabecera, llevando el proceso en relación con los familiares y/o allegados. Mas también puede y debe tener un protagonismo relevante el enfermero, contribuyendo con su mayor proximidad a enfermos y allegados a humanizar el proceso, alejándolo de la impersonalidad y la reificación que siempre lo amenazan, sobre todo cuando tiene lugar en un centro hospitalario.

Desafortunadamente, muchas veces, los familiares - los parientes y las personas afectadas- no disponen del tiempo o no poseen los recursos necesarios (entre ellos, la formación, la preparación) para hacerse cargo del enfermo terminal. Sin embargo, también muchas veces, esas deficiencias pueden superarse con la ayuda del médico de cabecera 51 . Los familiares suelen temer no saber enfrentarse a las situaciones y problemas que en el curso de la enfermedad puedan surgir. Temen no saber qué hacer y, en consecuencia, no cuidar adecuadamente al enfermo 52 . Temor acrecentado por tratarse de situaciones de gran intensidad emocional. Sin embargo,

\footnotetext{
47 Es más, como hemos venido insistiendo: "telling the truth is essential to the health of the doctorpatient relationship" (Higgs 2001, p. 92).

48 "Cada individuo muere de un modo similar a como ha vivido. Los modos en que uno responde habitualmente a las crisis cotidianas nos dan claves sobre el modo en que el cliente responderá a la crisis de la enfermedad o de la muerte" (Doka 2010, p. 119).

49 “Atender al moribundo" (Cabodevilla 2002, pp. 147-167).

50 "El apoyo de los demás" (Mittag 1996, pp. 112-116).

51 Médico, enfermero y no solo estos: el apoyo psicológico prestado por profesionales puede jugar un papel importante, tanto para el enfermo como para los acompañantes. Sobre la acción del psicólogo en las diferentes fases (diagnóstica, crónica, terminal) de la enfermedad mortal: Doka 2010.

52 “Claudicación familar" (Comisión Galega de Bioética 2012, p. 28).
} 
por lo general, con las indicaciones del médico de cabecera es posible prestar una atención adecuada ${ }^{53}$. Sin duda, con entrega y sacrificio personales,... que los familiares y allegados no siempre se encuentran en las condiciones materiales y psicológicas necesarias para realizarlos. De ahí, la necesidad de contar, ante estas situaciones, con el sistema y la organización sanitarios públicos adecuados ${ }^{54}$.

\subsection{Atención médica y aceptación del enfermo}

Por lo que hemos ido viendo, para el enfermo suele ser mejor pasar sus últimos tiempos en su entorno familiar, viviendo según sus convicciones ${ }^{55}$, con sus cosas y sus seres queridos. Y estos, en la medida que puedan dedicar un tiempo al enfermo, suelen también llevar mejor el trance y el duelo56.

Sintetizando, se trata de procurar al enfermo la mejor calidad de vida, evitando el dolor y el sufrimiento. Esto constituye la preocupación y el cometido fundamentales del facultativo: evitar o mitigar el dolor, mantener la calidad de vida ${ }^{57}$. Y de ello forma parte la comunicación con el paciente que suele manifestar, además de interés por saber cómo está, temor por la amenaza del dolor y preocupación por lo que le queda de vida. Son preocupaciones que también manifiestan los familiares, preguntándole al médico. Este debe transmitir confianza al enfermo y a los familiares, informando sin abrumar, con la prudencia requerida por el hecho de no ser la medicina una ciencia exacta ${ }^{58}$, ateniéndose a los hechos y permitiendo que el trans-

\footnotetext{
53 Víctor Mencía Seco y Rosa Díez Cagigal señalan: "Por si sirve de referencia, revisando nuestras estadísticas anuales, el porcentaje de pacientes llevados por nuestro equipo está, prácticamente cada año, ligeramente por encima del 80\%. Parece lógico que sea así: siempre habrá determinadas situaciones familiares, personales y sociales, que hagan conveniente, e incluso necesario, un ingreso en el hospital. Pero cuando el paciente recibe el apoyo suficiente en su domicilio, este porcentaje de personas que finalmente mueren en el hospital queda reducido al 20\%" (Mencía Seco \& Díez Cagigal 2006, pp. 84-85). Ahora bien, como poco antes registran, la inmensa mayoría de los enfermos fallecen en el hospital. Cosa que lamentan y critican, por no ser esa la preferencia también de la inmensa mayoría de los enfermos (Mencía Seco \& Díez Cagigal 2006, p. 84).

54 "Ayudar a morir bien es también objetivo institucional" (Comité de Bioética de Cataluña 2011, pp. 89-95). También: Comisión Galega de Bioética 2012, p. 24.

55 Para responder a las necesidades religiosas y/o espirituales, es menester la asistencia religiosa, la atención espiritual (Díez Ripollés, Santos Amaya \& Garrido de los Santos 2000, pp. 166-168).

56 A propósito de "morir en el hogar", apunta Mittag: "También para los parientes y amigos que cuidan de un enfermo de muerte tiene sus ventajas esta situación. La asistencia directa y cotidiana del enfermo ayuda a evitar sentimientos de culpabilidad y fantasías torturantes que a veces repercuten hondamente en el posterior proceso de luto" (Mittag 1996, p. 89).

57 "El sufrimiento causado por enfermedades es el azote impersonal e impune contra el que luchan los profesionales de la salud, los científicos y todos los servicios e instituciones sanitarias. Hay que seguir combatiendo el dolor, la muerte y la degradación física y psíquica que comportan. La muerte es inevitable, el sufrimiento en muchos casos no lo es. No es ético condenar al sufrimiento innecesario" (Boladeras 2009, p. 148).

58 Cfr. Comisión Galega de Bioética 2012, p. 20.
} 
curso del tiempo y la evolución de la enfermedad vayan haciendo a todos, incluido el paciente, más conscientes de la situación ${ }^{59}$. En que el trance sea lo más llevadero, y el desenlace lo mejor posible, tiene un papel crucial el facultativo, orientando correctamente la acción, evitando la obstinación terapéutica y limitando el esfuerzo terapéutico 60 .

\section{Reflexión final, algunas conclusiones}

No queremos acabar sin apuntar algunas conclusiones atinentes a cada uno de los cinco puntos tratados: 1 . decir la verdad; 2 . no querer saber; 3 . la verdad en el contexto y en el interior de la relación terapéutica; 4. información, comunicación; 5. comunicación, relación, atención.

1. Nuestras reflexiones versan sobre una cuestión resuelta en la teoría, pero persistente en la práctica. Tanto la legislación vigente como la bioética imperante (metodologías como el principialismo e instituciones como las comisiones y los comités de ética) marcan el deber de decir la verdad en la relación médico-paciente. Esta es además una obligación con fuerte apoyo en la moral occidental contemporánea.

En términos bioéticos, el principio de autonomía proporciona la regla, mientras que el principio de beneficencia (junto con el de no-maleficencia) sustenta las excepciones.

2. Sin embargo, cuando se trata de una enfermedad mortal, del morirse y la muerte, la verdad se resiente: hay una resistencia a decirla, basada grandemente en una resistencia a conocerla. Las causas se situarían en carencias vitales personales y en déficits culturales sociales que convierten la muerte en un tabú para el individuo y en la sociedad occidentales contemporáneos.

Entre las soluciones, que debe proporcionar la sociedad, subrayamos dos: disfrutar de una vida digna duradera y disponer de una formación, que incluya expresamente la filosofía, para afrontar la muerte.

3. Esta problemática psico-social excede el marco sanitario, pero no puede pasarse por alto, pues en el trance de la enfermedad mortal incide de lleno en la comunicación entre el médico y el paciente.

Desde el punto de vista del médico, la resistencia a decir la verdad se fundamenta, sobre todo, en el presunto beneficio del enfermo, aunque hay que considerar también otros factores como el rechazo a reconocer el fracaso y los límites de la

59 Desde 1969, constituye una referencia la secuenciación establecida por Elizabeth Kübler-Ross, su modelo fásico del morir: primera fase, negación y aislamiento; segunda fase, ira; tercera fase, pacto; cuarta fase, depresión; quinta fase, aceptación (Kübler-Ross 2010, pp. 59-177).

60 Comisión Galega de Bioética 2012, pp. 21-22. 
medicina e incluso el temor a que el paciente se vaya a otro médico. Faltar a la verdad en términos generales -aunque es necesario admitir graduaciones y excepciones- no resulta admisible en una relación médico-paciente que ya no es paternalista, sino que se inscribe en un marco contractualista moral y legal. Por otra parte, se hace necesario matizar el paternalismo, que enfocado como "parentalismo", como atención y cuidado a sujetos desprotegidos, frágiles y vulnerables, resulta provechoso como aproximación a la enfermedad terminal. Sin que esto signifique menoscabo de la autonomía y obliteración de la veracidad.

En la perspectiva del enfermo, el rechazo a la verdad, según cabe constatar, es, cuando menos, mucho menor del que habitualmente se presume. Además, es necesario asociar dicho temor a las consecuencias de la información: el hecho de convertirse en nuestra sociedad en un enfermo mortal y encaminarse hacia la muerte. El médico, el personal y el sistema sanitario, puede en este punto hacer mucho ofreciendo confianza, atención y cuidado para poder vivir dignamente hasta el final.

4. Los estudios muestran que la información al paciente sigue siendo un problema, pero afortunadamente un problema que se ha tomado en consideración y que se trabaja para darle solución.

¿Quién y cómo informar? Defendemos el protagonismo del médico de cabecera y una información graduada según las circunstancias, teniendo como umbral mínimo el consentimiento informado y como nivel óptimo la planificación conjunta de los cuidados.

5. El problema de la verdad debe ligarse a la cuestión de la atención. El temor a la verdad tiene mucho que ver con el miedo a la desatención, que va más allá del espacio médico, pues alcanza además a familiares y allegados. Nuevamente insistimos en el papel del médico de cabecera, junto con el personal sanitario, al lado ahora de los familiares y allegados. Subrayamos la conveniencia del domicilio como lugar para la atención médica, el acompañamiento al enfermo y la aceptación de la enfermedad. Y el papel del médico orientando la acción terapéutica encaminada a procurar al enfermo la mejor calidad de vida y a aliviar el dolor y el sufrimiento del moribundo.

6. Por último, volviendo al principio, insistimos en la importancia, la utilidad y la necesidad de realizar trabajos conjuntos, de abordar cuestiones desde ángulos diferentes, como la filosofía y la medicina.

\section{Referencias bibliográficas}

ARIÈs, Ph. (1975): Essais sur l'histoire de la mort en Occident du Moyen Age à nos jours, Paris, Éditions du Seuil.

Beauchamp, T. L. \& Childress, J. F. (2002): Princípios de ética biomédica, trad. port. Luciana Pudenzi, São Paulo, Edições Loyola. 
Boladeras, M. (2009): El derecho a no sufrir. Argumentos para la legalización de la eutanasia, Barcelona, los libros del lince.

Cabodevilla, I. (2002): Vivir y morir conscientemente, Bilbao, Desclée de Brouwer.

Childress, J. \& Siegler, M. (2001): "Metaphors and Models of Doctor-Patient Relationships: Their Implications for Autonomy", en Mappes, Th. A. \& DeGrazia, D., Biomedical Ethics, New York, Mac Graw-Hill, pp. 71-80.

Comisión Galega de Bioética (2012): Os coidados ao final da vida. Documento de recomendacións, Santiago de Compostela, Xunta de Galicia.

Comité de Bioética de Cataluña (2011): Recomendaciones a los profesionales sanitarios para la atención a los enfermos al final de la vida, Barcelona, Generalitat de Catalunya-Fundació Víctor Grífols i Lucas.

Díez Ripollés, J.L., SANTOS Amaya, I.M. y GARrido de los SAntos, Ma . J., (2000): La atención a los enfermos terminales. Una investigación multidisciplinar, Valencia, tirant lo blanch.

DoKA, K.J. (2010): Psicoterapia para enfermos en riesgo vital, trad. cast. Jasone Aldekoa, Bilbao, Desclée de Brouwer.

ENGELHARDT, H.T. (1995): Los fundamentos de la bioética, trad. cast. Isidro Arias, Gonzalo Hernández \& Olga Domínguez, Barcelona-Buenos Aires-México, Paidós.

GIL, F. (2005): "Mors certa, hora incerta”, en Gil, F., Acentos, Lisboa, Imprensa Nacional-Casa da Moeda, pp. 291-304.

Gondar Portasany, M. (1989): Romeiros do Alén. Antropoloxía da morte en Galicia, Vigo, Xerais.

Gracia, D. (2008): "Primum non nocere. O concepto de "dano" e os seus problemas”, Encrucillada. Revista Galega de Pensamento Cristián, 159, pp. 5-17.

Higgs, R. (2001): "On Telling Patients the Truth", en Mappes, Th. A. \& DeGrazia, D., Biomedical Ethics, New York, Mac Graw-Hill, pp. 87-93.

Hottois, G. y Missa, J.-N. (2005): Nova enciclopedia de bioética, trad. gal. Luís G. Soto \& Tareixa Roca, Santiago de Compostela, Universidade de Santiago de Compostela.

Imber, J. B. (2008): Trusting Doctors. The Decline of Moral Authority in American Medicine, Princeton \& Oxford, Princeton University Press.

KANT, I. (1986): "Sobre un presunto derecho a mentir por filantropía", en Kant, I., Teoría y práctica, trad. cast. Juan Miguel Palacios, Madrid, Tecnos, pp. 61-68.

Kant, I. (1988): Fundamentação da Metafísica dos Costumes, trad. port. Paulo Quintela, Lisboa, Edições 70.

KüBler-Ross, E. (2010): Sobre la muerte y los moribundos, trad. cast. Neri Daurella, Barcelona, Debolsillo.

Lora, P. de \& Gascón, M. (2008): Bioética. Principios, desafios, debates, Madrid, Alianza. 
Marinoff, L. (2003): Más Platón y menos prozac, trad. cast. Borja Folch, Madrid, Suma de las Letras.

MARQues, M. S. (2010): "Uma sombra o precede. Lei de morte, hospitalidade e cuidados de suporte", Alicerces, III (3), pp. 147-165.

Mencía Seco, V.R. \& Díez Cagigal, R. (2006): Manejo del enfermo terminal en su domicilio. Actitudes y aptitudes de los profesionales ante la enfermedad, la agonía y la muerte, Alcalá La Real (Jaén), Formación Alcalá.

Mill, J.S. (1981): Sobre la libertad, trad. cast. Pablo de Azcárate, Madrid, Alianza. Mittag, O. (1996): Asistencia práctica para enfermos terminales, trad. cast. Claudio Gancho, Barcelona, Herder.

Nilstun, T. (2005): "Paternalismo", en Hottois, G. \& Missa, J.-N. (dirs.), Nova enciclopedia de bioética, Santiago de Compostela, Universidade de Santiago de Compostela, pp. 684-686.

PARIZEAU, M.-H. (2005): "Verdade (dicir ao doente a)", en Hottois, G. \& Missa, J.N. (dirs.), Nova enciclopedia de bioética, Santiago de Compostela, Universidade de Santiago de Compostela, pp. 907-910.

Pose, C. (2009): Lo bueno y lo mejor. Introducción a la bioética médica, Madrid, Triacastela.

SÁdABA, J. (2009): La vida buena. Cómo conquistar nuestra felicidad, Barcelona, Península.

SÁdABA, J. (2012): No sufras más. La felicidad en la vida cotidiana, Barcelona, Península.

Simón, P. y JúdEZ, J. (2004): “Consentimiento informado”, en Gracia, D. \& Júdez, J. (eds.), Ética en la práctica clínica, Madrid, Triacastela, pp. 33-54.

Tugendhat, E. (2010): Lecciones de ética, trad. cast. Luis Román Rabanaque, Barcelona, Gedisa.

WinOKUR, S. (2012): “¿Por qué eludimos la verdad con los pacientes?”, Diario Médico, 12 de setiembre de 2012, p. 2.

\section{Luís G. Soto}

Facultade de Filosofía

Universidade de Santiago de Compostela

luisg.soto@usc.es

Carlos Sánchez Fernández de la Vega

Centro de Saúde de Fingoi-Lugo

camesues@gmail.com 